\title{
Padrão de Distribuição da Calda Produzida pela Ponta de PulverizaÇão do TIPo J ATO Plano (8002) EM Função do ESPAÇAMENTO ENTRE BICOS ${ }^{1}$
}

\author{
Spray Distribution Pattern of Flat Fan Tip (8002) at Different Spacing Between Nozzles
}

\author{
LACERDA, A.L.S. ${ }^{2}$
}

\begin{abstract}
RESUMO - O presente trabalho foi desenvolvido no laboratório de Engenharia do Departamento de Engenharia Rural da Escola Superior de Agricultura "Luiz de Queiroz" (USP/ESALQ), Piracicaba-SP, com o objetivo de estudar o padrão de distribuição da ponta de pulverização do tipo jato plano (leque) 8002, em função das distâncias entre os bicos. Para isso, foram colocados 10 bicos sobre uma mesa de prova, um de cada vez, a uma altura fixa de $45 \mathrm{~cm}$, nos espaçamentos de 10 a $80 \mathrm{~cm}$. Os volumes coletados em cada uma das canaletas serviram para calcular o coeficiente de variação e iniciar o estudo do espaçamento adequado. Após os testes realizados, pôde-se concluir que o bico tipo jato plano 8002 mostrou melhor distribuição em espaçamento de $50 \mathrm{~cm}$, com menor coeficiente de variação $(6,88 \%)$ a uma altura fixa de $45 \mathrm{~cm}$ e pressão regulada a $279,3 \mathrm{Kpa}$, registrada no manômetro.
\end{abstract}

Palavras-chave: variabilidade, espaçamento, manômetro.

\begin{abstract}
This work was carried out at the Department of Agricultural Engineering, Escola Superior de Agricultura "Luiz de Queiroz" (USP/ESALQ), Piracicaba-SP, Brazil. The objective of this research was to study the spray pattern of the flat fan tip (8002) at different spacings between nozzles. Thus, ten nozzles were placed on a tilted foil, one at a time, at a fixed height of $45 \mathrm{~cm}$, with spacings varying from 10 to $80 \mathrm{~cm}$. Nozzle output was collected separately from each nozzle and used to calculate the variation coefficient and determine the more appropriate spacing between nozzles. It was concluded that, $50 \mathrm{~cm}$-spaced flat fan tips (8002) showed the best spray pattern with a smaller variation coefficient at a fixed height of $45 \mathrm{~cm}$ and pressure regulated at $279.3 \mathrm{kPa}$ registered in the manometer.
\end{abstract}

Key words: variability spacing, manometer.

\section{INTRODUÇÃO}

Um dos fatores que mais afetam a eficácia de um defensivo agrícola é a distribuição da pulverização. A simples determinação do volume de calda a ser aplicado em uma determinada área não é suficiente para avaliar a qualidade da aplicação ou do defensivo agrícola, pois esta não depende somente da vazão, mas também de como é que este defensivo está sendo distribuído na área, para que se obtenha máxima eficácia com o mínimo de desperdício e de contaminação do meio ambiente.
O bico é o componente mais importante no equipamento de pulverização, uma vez que é o responsável pela formação, pelo tamanho e pela distribuição das gotas na área desejada. Cada tipo de bico pulverizador possui um perfil de distribuição volumétrica característico. Esse perfil tem grande importância na determinação do melhor espaçamento dos bicos entre si, numa barra de pulverizador.

Segundo Sartori (1975), o perfil ideal de distribuição volumétrica dos bicos deve possuir características achatadas ou levemente

Recebido para publicação em 1/6/2001 e na forma revisada em 17/12/2001.

2 Eng.-Agrônomo, Doutorando em Agronomia no Dep. de Produção Vegetal - ESALQ/USP, Av. Pádua Dias, 11, Cx. Postal 9, 13417-950 Piracicaba-SP, <alslacer@carpa.ciagri.usp.br>. 
triangular, suave e sem saltos, a fim de possibilitar na área de aplicação uma distribuição uniforme quando sobrepor os bicos.

A tendência atual de se empregar pulverização em volume e pressões mais reduzidas torna a seleção dos bicos pulverizadores bastante crítica. Com isso, a uniformidade do defensivo agrícola fitossanitário sobre o alvo constitui uma condição essencial para alcançar bons resultados. Assim, a escolha incorreta dos bicos constitui-se numa das principais causas responsáveis por fracassos na aplicação de defensivos (Matuo, 1982).

Mesmo sendo o bico pulverizador o elemento fundamental na distribuição de agroquímicos, segundo Reed \& Ferraza (1984), existe suficiente evidência de que ele também é o componente que menos recebe atenção e controle durante a vida útil do equipamento.

O conhecimento da distribuição quantitativa da calda pulverizada ao longo da faixa de deposição é, portanto, de grande importância no estudo da pulverização (Matuo, 1982).

O presente trabalho teve como objetivo conhecer o perfil de distribuição de um bico de pulverização da tipo jato plano (leque) em função das distâncias entre si.

\section{MATERIAL E MÉTODOS}

O trabalho foi desenvolvido no laboratório de Engenharia da Escola Superior de Agricultura "Luiz de Queiroz", em Piracicaba, no Departamento de Engenharia Rural.

O estudo do perfil de distribuição do bico hidráulico foi efetuado numa mesa de prova que atende às normas da ABNT - NBR 13769 (1997), constituída por canaletas espaçadas de $50 \mathrm{~mm}$ entre si, tendo um coletor (béquer) graduado em cada canaleta. Os volumes de água pulverizados pelos bicos montados em um circuito de pressão regulado para $279,3 \mathrm{KPa}$, registrado no manômetro, foram coletados nos béqueres durante o tempo suficiente para que pelo menos um dos recipientes atingisse em torno de $90 \%$ de sua máxima capacidade.

O bico utilizado foi do tipo jato plano (leque) 8002, da marca Magno, que tem como característica uma ponta jato plano (leque) convencional, ângulo do jato de $80^{\circ}$, vazão de $0,76 \mathrm{~L} \mathrm{~min}^{-1} \mathrm{a}$ pressão de 279,3 KPa e espaçamento recomendado entre bicos de $50 \mathrm{~cm}$ pelo fabricante.

Foram formados dois grupos de 10 bicos, ambos colocados sobre a mesa de prova, um bico por vez na canaleta central, a uma altura fixa de $45 \mathrm{~cm}$. O volume coletado nos béqueres serviu para selecionar um dos grupos de bicos, através da menor média do coeficiente de variação, calculado pela fórmula: $\mathrm{CV}=$ desviopadrão/volume médio x 100, e iniciar o estudo do espaçamento adequado. Com o volume médio encontrado, no grupo inicialmente selecionado, elaborou-se o gráfico de histograma representativo da faixa de deposição e avaliou-se a influência dos espaçamentos de 10 a $80 \mathrm{~cm}$ na uniformidade de distribuição (CV), por meio da sobreposição de pelo menos 36 canaletas e desprezando-se os extremos onde não houve sobreposição.

\section{RESULTADOS E DISCUSSÃO}

\section{Seleção dos bicos}

Os grupos 1 e 2 apresentaram CV médio de 55,6 e $53,2 \%$, respectivamente, sendo este último selecionado para o estudo em questão, conforme pode ser visto na Figura 1 e no Quadro 1.

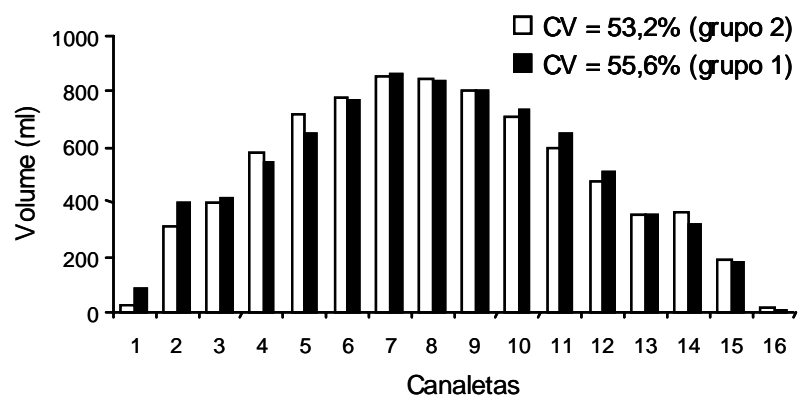

Figura 1 - Coeficientes de variação do volume médio do líquido de 10 bicos (grupos 1 e 2) pulverizados a pressão de 279,3 $\mathrm{KPa}$, altura de $45 \mathrm{~cm}$, coletados em canaletas espaçadas de $50 \mathrm{~mm}$.

\section{Perfis de distribuição e faixa de deposição}

Os padrões de deposição dos bicos nos espaçamentos de 10 a $80 \mathrm{~cm}$ são apresentados na Figura 2. Os gráficos expressam na abcissa as canaletas da mesa de prova e na ordenada 
os volumes de aplicação. Pode-se notar pelas figuras que, à medida que se aumentam os espaçamentos dos bicos, principalmente acima de $50 \mathrm{~cm}$, a distribuição fica desuniforme, fazendo com que a sobreposição se torne quase impossivel.

Quadro 1 - Vazão média, desvio-padrão e coeficiente de variação obtidos dos volumes médios coletados nas canaletas para os grupos 1 e 2

\begin{tabular}{|c|c|c|}
\hline Canaletas & Grupo 1 & Grupo 2 \\
\hline 15 & 0 & 0 \\
\hline 14 & 0 & 0 \\
\hline 13 & 0 & 0 \\
\hline 12 & 0 & 0 \\
\hline 11 & 0 & 0 \\
\hline 10 & 0 & 0 \\
\hline 9 & 0 & 0 \\
\hline 8 & 0 & 0 \\
\hline 7 & 27 & 88 \\
\hline 6 & 309 & 400 \\
\hline 5 & 400 & 418 \\
\hline 4 & 580 & 550 \\
\hline 3 & 715 & 649 \\
\hline 2 & 780 & 773 \\
\hline 1 & 859 & 866 \\
\hline 0 & 846 & 839 \\
\hline 1 & 800 & 805 \\
\hline 2 & 713 & 732 \\
\hline 3 & 600 & 647 \\
\hline 4 & 480 & 507 \\
\hline 5 & 349 & 354 \\
\hline 6 & 366 & 322 \\
\hline 7 & 190 & 180 \\
\hline 8 & 13 & 10 \\
\hline 9 & 0 & 0 \\
\hline 10 & 0 & 0 \\
\hline 11 & 0 & 0 \\
\hline 12 & 0 & 0 \\
\hline 13 & 0 & 0 \\
\hline 14 & 0 & 0 \\
\hline 15 & 0 & 0 \\
\hline Média & 501,68 & 508,75 \\
\hline Desvio & 279,23 & 270,47 \\
\hline $\mathrm{CV}$ & 55,65 & 53,16 \\
\hline
\end{tabular}

Para espaçamento de $10 \mathrm{~cm}$, o padrão de distribuição tem forma triangular, de 20 a $50 \mathrm{~cm}$, tende a se apresentar mais achatado (trapezoidal) e espaçamento de 60,70 e $80 \mathrm{~cm}$, de forma irregular.

O ideal seria uma distribuição achatada ou levemente triangular, suave e sem saltos, para possibilitar na área de aplicação uma distribuição uniforme, quando se sobreporem os bicos. Segundo Sartori (1975), a distribuição raramente é uniforme, sendo mais comum as distribuições parabólicas e triangulares e menos freqüentes as trapezoidais.

A grande variação na distribuição indica desuniformidade de volume aplicado, podendo isso significar subdosagem ou dosagem excessiva na área de aplicação, comprometendo a eficácia do defensivo agrícola.

Também se pode notar que nas extremidades da faixa de aplicação ocorre diminuição do líquido pulverizado, sendo máxima sob o eixo dos bicos, concordando com Balastreire (1970).

\section{Coeficiente de variação}

Conforme pode ser visto na Figura 3 , os espaçamentos de 30,40 e $50 \mathrm{~cm}$ obtiveram coeficientes de variação médios de 7,76, 7,42 e $6,88 \%$, respectivamente.

Considerando o CV limite de $7 \%$ para que uma distribuição seja extremamente uniforme, segundo a fábrica de bicos e acessórios Spraying Systens (1998) e a entidade de pesquisa Biologische Bundesanstalt FûrlandUnd Forstwrts-Chaft Bundesrepublik Deustschaland, citada por Furlanetti (1995), neste estudo somente o espaçamento de $50 \mathrm{~cm}$ obteve coeficiente de variação aceitável.

O melhor espaçamento para esse tipo de bico é o de $50 \mathrm{~cm}$ de distância entre si, concordando com o recomendado pelo fabricante, pois foi o que obteve o menor coeficiente de variação $(6,88 \%)$. Isso quer dizer que há melhor distribuição e sobreposição dos bicos a uma distância de $50 \mathrm{~cm}$.

À medida que se diminui o espaçamento abaixo de $30 \mathrm{~cm}$ e acima de $50 \mathrm{~cm}$ entre os bicos, piora-se a uniformidade de aplicação, principalmente para os espaçamentos acima de $50 \mathrm{~cm}$. 

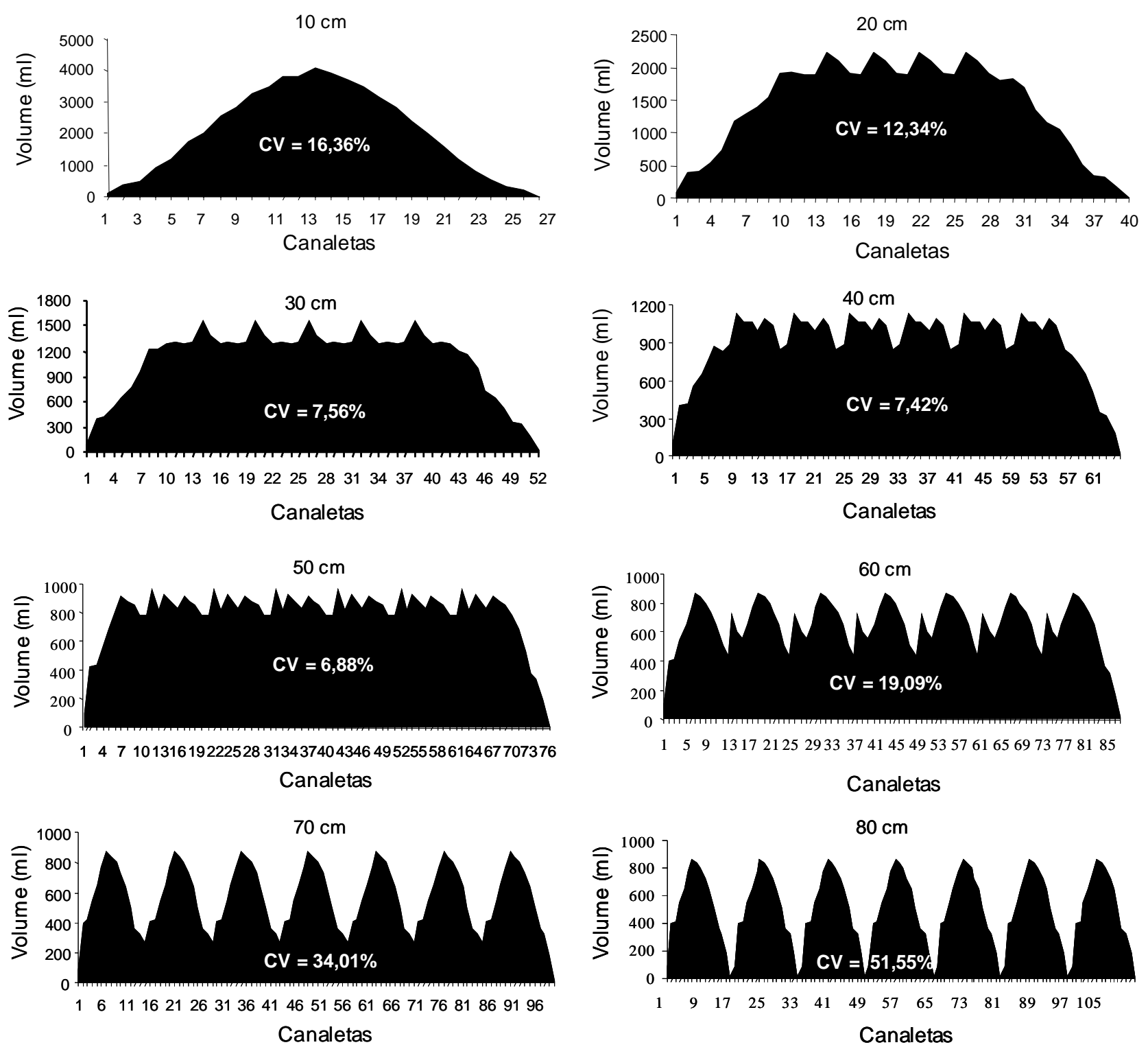

Figura 2 - Distribuição do volume médio do líquido pulverizado nas canaletas e respectivo coeficiente de variação do bico Magno 8002, trabalhando à pressão de $279,3 \mathrm{KPa}$, a uma altura de $45 \mathrm{~cm}$ em relação ao alvo, para os espaçamentos entre os bicos de 10, 20, 30, 40, 50, 60, 70 e $80 \mathrm{~cm}$.

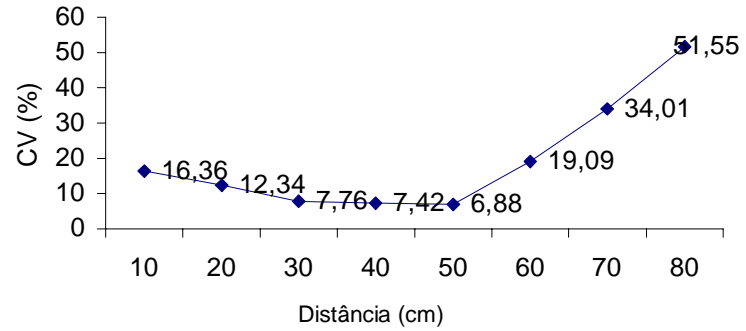

Figura 3 - Coeficiente de variação encontrado em função dos espaçamentos de 10 a $80 \mathrm{~cm}$ em bico do tipo jato plano (leque).
Os CVs médios obtidos para espaçamentos de 60, 70 e $80 \mathrm{~cm}$ foram de 19,09, 34,01 e $51,55 \%$, tornando a sobreposição dos bicos quase impossivel em termos de qualidade de distribuição do líquido ou defensivo agrícola pulverizado, ocasionando na área grande irregularidade (grandes variações) da distribuição dos volumes aplicados e conseqüente deposição irregular (pontos com grande volume depositado e pontos com baixa deposição) (Sartori, 1975). 


\section{CONCLUSÕES}

O bico do tipo jato plano 8002 da marca Magno apresentou melhor distribuição no espaçamento de $50 \mathrm{~cm}$, com menor coeficiente de variação $(6,88 \%)$ a uma altura fixa de $45 \mathrm{~cm}$ e pressão regulada a 279,3 Kpa, registrada no manômetro.

\section{LITERATURA CITADA}

ASSOCIAÇÃO BRASILEIRA DE NORMAS TÉCNICAS - ABNT. Bicos de pulverização agrícola: métodos de ensaio - NBR 13769. Rio de Janeiro: 1997. 16p.

BALASTREIRE, L.A. Bicos pulverizadores: estudo da faixa de deposição. Piracicaba: ESALQ, 1970. 81p. Tese (Doutorado em Fitotecnia) - Escola Superior de Agricultura “Luiz de Queiroz”, 1970.

FURLANETTI, A.C. Vazão e deposição de bicos pulverizadores de jato em leque. Botucatu: UNESP, 1995. 123p. Dissertação (Mestrado em Fitotecnia) - Universidade Estadual Paulista Júlio de Mesquita Filho, 1995.
MATUO, T. Tecnologia de aplicação de defensivos. In: GRAZIANO NETO, F., ed. Uso de agrotóxico. São Paulo: Agroedições, 1982. p.103-106.

PERECIN, D.; PERESSIN, V.A; MATUO, T.; BARBOSA, J.C.; PIO , L.C.; BRAZ, B.A. Padrões de distribuições obtidos com bicos Twinjet, em função da altura e do espaçamento entre bicos. Eng. Agríc., v.14, p.19-30, 1994.

REED, T.; FERRAZZA, J. Wear life of agricultural nozzles. St Joseph: ASAE, 1984. 19p. (ASAE Paper, AA84-001).

SARTORI, S. Considerações a respeito da aplicação de defensivos por via líquida. Pompéia: Máquinas Agrícolas Jacto, 1975. 30p.

SARTORI, S. Pulverizadores para aplicação terrestre tratorizado. In: SIMPÓSIO BRASILEIRO SOBRE TECNOLOGIA DE APLICAÇÃO DE DEFENSIVOS AGRÍCOLAS, 1, 1985, Jaboticabal. Anais... Jaboticabal: FUNEP, 1985. p.47-79. 1985.

SPRAYING SYSTEMS CO. TeeJ et: Defensivo agrícola de pulverização para a agricultura. Wheaton: 1998. 80p. (Cat. 44M-P). 